\title{
ORIGINAL RESEARCH \\ What's in Your Mouth? The CT Appearance of Comestible Intraoral Foreign Bodies
}

M. McDermott

B.F. Branstetter IV

E.J. Escott
BACKGROUND AND PURPOSE: Comestible or chewable intraoral foreign bodies (IOFB), such as candies, gum, and chewing tobacco, are seen incidentally on many CT scans of the head and neck. If these foreign bodies are misinterpreted as pathology, patients may be subjected to unnecessary distress or unneeded radiation from additional imaging. The purpose of this study was to characterize the CT appearance of comestible IOFBs and to find characteristics that distinguish them from true pathology.

MATERIALS AND METHODS: With institutional review board approval, 30 patients who were already scheduled to undergo CT examinations of the head and neck were enrolled in this study. Nine typical IOFBs with different physical characteristics were selected for inclusion. Each patient placed 1 IOFB in his or her mouth before the initiation of the routine clinical scan. The resulting scans were evaluated by 2 head and neck radiologists. In vivo and ex vivo attenuation measurements were obtained for each IOFB.

RESULTS: The attenuation of comestible IOFBs ranged from 184 to 475 Hounsfield units. Large, hard IOFBs were most easily distinguished from mucosal lesions, but might be mistaken for odontogenic or bone tumors. Small, hard IOFBs could be mistaken for calculi, tooth fragments, or enhancing vessels. Soft IOFBs generally had more confusing configurations and more heterogeneous densities and, thus, might be mistaken for enhancing mucosal lesions. Foci of gas were often identified within chewable IOFBs, mimicking an abscess. Because all of the IOFBs had higher densities than soft tissue, they could all be mistaken for calcified, enhancing, or bony lesions.

CONCLUSION: Radiologists frequently encounter IOFBs on CT examinations of the head and neck. Familiarity with the expected appearance of these incidental pseudolesions is important to prevent misdiagnosis as a true pathologic process.
$\mathbf{T}$ he presence of an intraoral foreign body (IOFB) on a CT examination of the head and neck may result in a surprisingly difficult diagnostic challenge. When patients undergo imaging that includes the oral cavity, they are routinely asked to remove foreign bodies such as dentures, chewing gum, and chewing tobacco. Occasionally, patients do not comply with this request, and the unexpected IOFB can masquerade as true pathology of the oral cavity. Such a misdiagnosis can result in additional scans with unnecessary irradiation, distraction from more relevant pathology, and unnecessary patient distress.

Previous studies on the radiographic appearance of comestible foreign bodies have focused on the abdomen, including reports of soft candies mimicking abdominal calcifications, ${ }^{1}$ and on the high attenuation of ingested chewing gum in the abdominal cavity. ${ }^{2}$ Studies regarding foreign bodies in the orbit tend to focus on the appearance of wood, metal, glass, or other penetrating objects. ${ }^{3-5}$ Previous literature on IOFBs has been restricted to metallic piercings, chronically retained food, or surgical material. ${ }^{6,7}$ There is currently no literature on the CT appearance of comestible IOFBs. The purpose of this study was to characterize the CT appearance of comestible IOFBs and to find characteristics that distinguish them from true pathology.

Received February 7, 2008; accepted after revision March 7

From the Departments of Radiology (M.M., B.F.B., E.J.E.) and Otolaryngology (B.F.B.), University of Pittsburgh School of Medicine, Pittsburgh, Pa.

Please address correspondence to Barton F. Branstetter IV, Department of Radiology, PUH Room D132, 200 Lothrop St, Pittsburgh, PA 15213; E-mail: bfb1@pitt.edu

DOI 10.3174/ajnr.A1127

\section{Materials and Methods}

\section{Patient Selection}

The University of Pittsburgh Institutional Review Board approved this prospective study, and written informed consent was obtained from each patient. Thirty consecutive patients (20 women and 10 men with an average age of 47 years and an age range of 19-69 years) who were scheduled to undergo CT scans of the head and neck that included the oral cavity were enrolled in the study to obtain 27 diagnostic quality scans of the oral cavity. Exclusion criteria were as follows: 1 ) age $<18$ years; 2) personal history of diabetes; 3 ) symptoms or history suggestive of oral pathology; or 4) extensive streak artifact through the oral cavity. Three patients were randomly assigned to each of the 9 different IOFBs. The remaining 3 patients were enrolled to substitute for patients with uninterpretable scans due to artifact from dental amalgam (exclusion criterion 4).

\section{Categories of IOFB}

To include a broad spectrum of different IOFBs in this study, several categories of IOFB were defined (Table 1). Categories were selected on the basis of consistency, size, and shape. The 2 main classes of IOFBs were hard (or solid) and soft (or chewable). The hard IOFBs were further divided into "large candies" and "small candies." The soft IOFBs were divided into "soft candies" and "gums." Both hardshelled and soft gums were included.

\section{Imaging Parameters}

Ex vivo CT of the IOFBs was performed to obtain baseline appearance and radiodensity measurements. Radiodensity was measured with a maximal circular region of interest on the largest cross-section.

Before in vivo scanning, patients were provided with the IOFB and told to place it in their mouth in a position of their choice. Patients 


\begin{tabular}{|c|c|c|}
\hline Category & Subcategory & Name \\
\hline \multirow[t]{2}{*}{ Hard IOFBs } & Large candies & $\begin{array}{l}\text { Jawbreaker (Ferrara Pan Candy Company, } \\
\text { Forest Park III) } \\
\text { Lemonhead (Ferrara Pan Candy Company, } \\
\text { Forest Park III) } \\
\text { Werther's Originals (Storck Industry, } \\
\text { Chicago, III) }\end{array}$ \\
\hline & Small candies & $\begin{array}{l}\text { tic tacs (Ferrero, Somerset, NJ) } \\
\text { Lifesaver (Wrigley, Peoria, III) }\end{array}$ \\
\hline \multirow[t]{2}{*}{ Soft IOFBs } & Chewables & $\begin{array}{l}\text { Tootsie Roll (Tootsie Roll Industries, } \\
\text { Chicago, III) } \\
\text { Starburst (Mars, Vernon, Calif) }\end{array}$ \\
\hline & Gums & $\begin{array}{l}\text { Bubble Yum (Hershey, Hershey, Pa) } \\
\text { Eclipse (Wrigley, Peoria, III) }\end{array}$ \\
\hline
\end{tabular}

Note:-10FB indicates intraoral foreign body.

\begin{tabular}{|c|c|c|}
\hline IOFB & Ex Vivo Density & Average In Vivo Density \\
\hline Jawbreaker & 418 & 475 \\
\hline Lemonhead & 253 & 323 \\
\hline Werther's & 321 & 411 \\
\hline Lifesaver & 386 & 464 \\
\hline tic tac & 393 & 335 \\
\hline Starburst & 259 & 347 \\
\hline Tootsie Roll & 260 & 357 \\
\hline Bubble Yum & 184 & 290 \\
\hline Eclipse gum & 360 & 383 \\
\hline
\end{tabular}

Note:-IOFB indicates intraoral foreign body.

were reminded not to chew during the scan. The routine clinical examination was then performed without additional modification. Each clinical radiology report made specific mention of the IOFB to avoid misinterpretation.

\section{Image Interpretation}

The scans were reviewed in consensus by 2 fellowship-trained Certificate of Added Qualification-certified neuroradiologists with 8 and 15 years of experience in head and neck imaging (B.F.B. and E.J.E.), who attempted to identify imaging patterns specific for IOFBs. Circular region of interest radiodensity measurements were obtained at the level of the largest cross-section and compared with the ex vivo densities.

\section{Results}

\section{General Characteristics}

The attenuation of IOFBs ranged from 184 to 475 Hounsfield units (Table 2). Thus, IOFBs were all substantially more radiodense than soft tissue. Most were slightly more radiodense in vivo than ex vivo.

Hard IOFBs retained their shape and size on the in vivo portion of the experiment. Hard IOFBs had, in general, greater attenuation compared with soft IOFBs. Hard IOFBs were significantly easier to appreciate on the scan compared with the soft IOFBs due to their size and retention of a characteristic shape. Another common feature was the appearance of collapsed mucosa nearly all the way around the hard IOFB, with an inconstant small air pocket on one side. The main variability among the hard candies was the internal texture: laminated, heterogeneous, and uniform densities were encountered. Large candies were least likely to be confused with

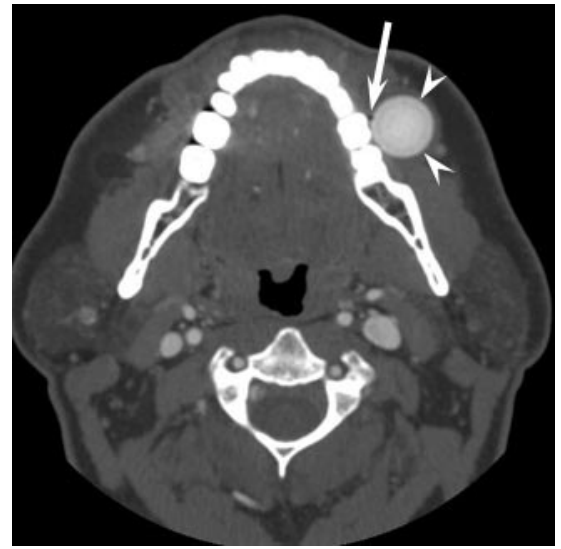

Fig 1. Large hard candy. Contrast-enhanced axial CT demonstrates a perfectly round attenuated mass (arrowheads) in the left lower gingivo-buccal sulcus. Note the laminated appearance of the mass. The surrounding mucosa is completely collapsed on the mass, except for a tiny locule of air (arrow)

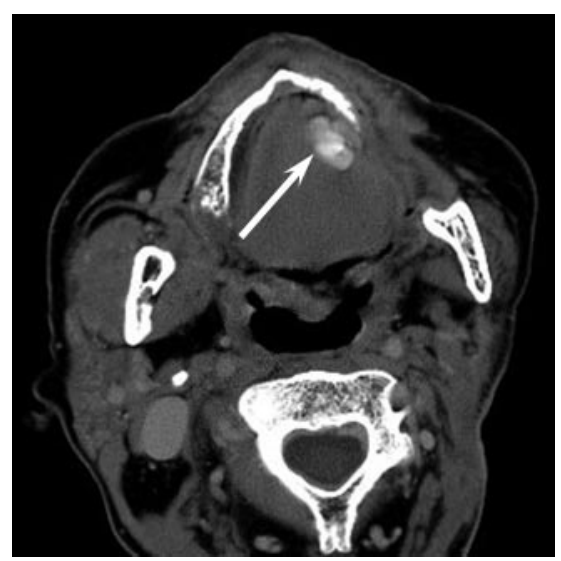

Fig 2. Small candy with motion artifact. Contrast-enhanced axial CT shows a small, attenuated focus (arrow) in the left floor of mouth, with seemingly variable attenuation. The heterogeneity is actually the result of motion artifact.

pathology but might have been mistaken for an osteoma or other bony excrescence (Fig 1). Small candies were prone to motion artifact, which increased the likelihood of misdiagnosis (Fig 2). Movement of IOFBs between acquisition sequences was more probable with small candies.

Soft IOFBs were more difficult to appreciate on the scan compared with the hard candies. Because these IOFBs are chewable, they may lack a characteristic shape or internal texture that makes them identifiable. Characteristics of the soft IOFBs included lower average radiodensity, amorphous shape, and predictable location. Because the soft IOFBs were typically chewed before the scan, the patients tended to hold the IOFB against the teeth or within mucosal recesses during the scan. Because the soft IOFBs molded to the shape of the underlying mucosal surface, they could easily be mistaken for enhancing mucosal lesions (Fig 3).

\section{Specific Characteristics}

Jawbreakers were the largest IOFBs tested and had a spherical configuration that facilitated identification as a foreign body. They have a laminated appearance with concentric circles of variable radiodensity throughout the internal architecture. Similar to the other hard IOFBs, jawbreakers showed col- 


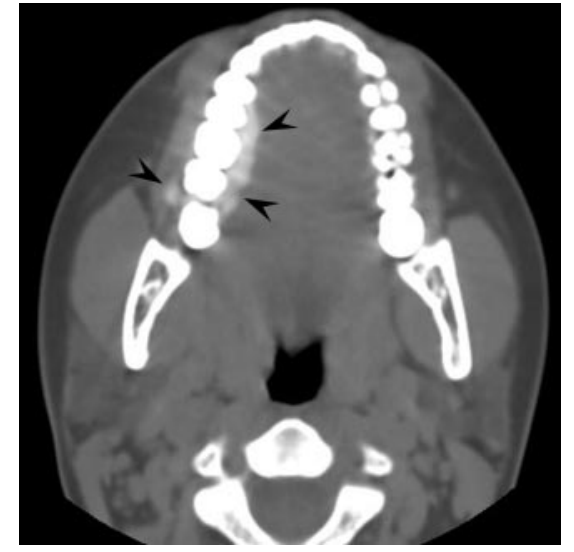

Fig 3. Soft foreign body. Unenhanced axial CT demonstrates an attenuated, irregular mass (arrowheads) along the alveolar ridge. On an enhanced scan, this might be mistaken for an enhancing mucosal malignancy.

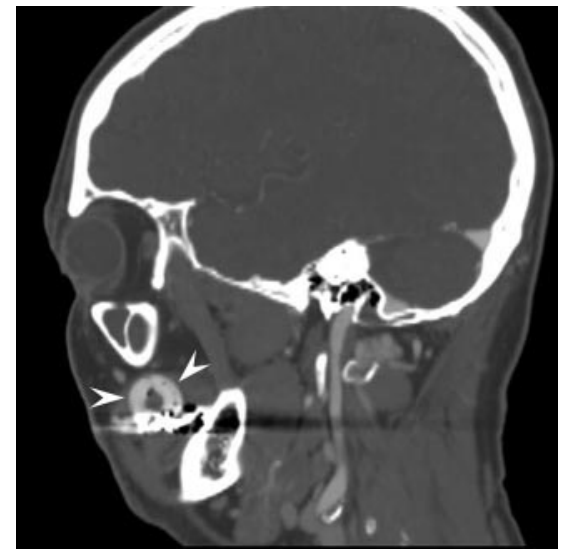

Fig 4. Hard candy. Sagittal reconstruction from enhanced CT shows a curvilinear attenuation (arrowheads) in the oral cavity representing a ring-shaped intraoral foreign body. The attenuation is similar to that of the contrast-enhanced vessels, and the inferior aspect of the foreign body is obscured by dental amalgam, such that the foreign body might be mistaken for an enlarged vessel or vascular abnormality.

lapsed mucosa almost all around the candy with a small air locule sometimes present on one side (Fig 1).

Lemonheads also had a spherical shape easily identified on the scan. The outer shell was of higher attenuation than the interior, which was of heterogeneous attenuation. Like the other hard IOFBs, Lemonheads were surrounded by collapsed mucosa with an inconstant small air locule.

When imaged along their length, Werther's Originals appeared circular, but when imaged in cross-section, the slight indentation in the center of the candy could be appreciated. The entire candy was homogenously very attenuating.

When imaged in their entirety, Lifesavers had a recognizable ring configuration. However, if portions of the candy were obscured by dental artifact or were crushed by chewing, the remaining semicircle could be mistaken for an enlarged vessel or displaced dental hardware (Fig 4).

Despite a seemingly characteristic shape, tic tacs were the most easily misidentified of the hard IOFBs. If held under the tongue, they could be confused with calculi in the Wharton duct (Fig 5). In addition, depending on the angle and position, a tic tac could be mistaken for a calcified tumor, a displaced tooth, or a torus malformation of the jaw. Motion artifact was

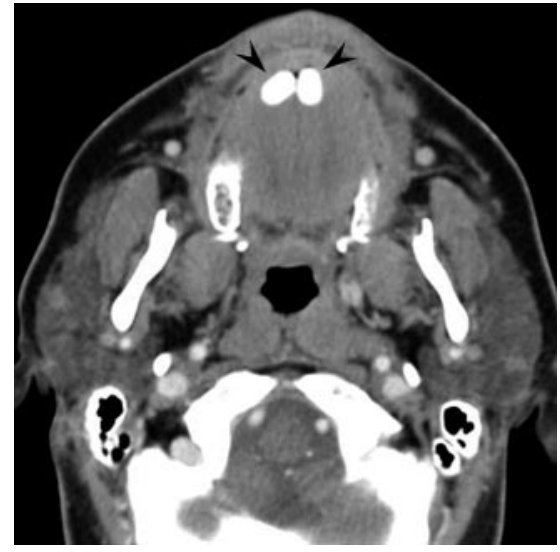

Fig 5. Small candies. Enhanced axial CT demonstrates 2 rounded densities (arrowheads) in the anterior oral cavity that might be mistaken for sialoliths impacted at the punctum of the Wharton duct.

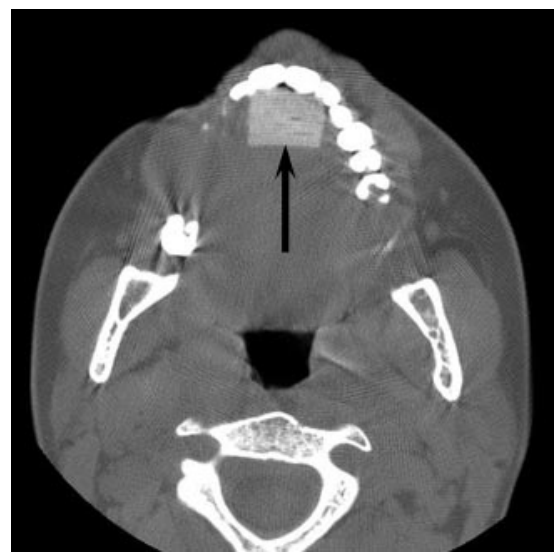

Fig 6. Soft intraoral foreign body. Unenhanced axial CT shows a rectangular foreign body (arrow) in the anterior oral cavity. With this configuration, the foreign body might be mistaken for an implant.

more common with tic tacs and made the morphology harder to discern, making misidentification more probable (Fig 2).

Starbursts typically had heterogeneous internal radiodensity. When not chewed, the Starburst is similar to hard IOFBs in that is has a characteristic size and shape. In this configuration, the Starburst may be confused with a dental prosthesis or silastic block (Fig 6). When chewed, the candy loses its square configuration and size, so if it is found in a mucosal recess, it may be mistaken for an enhancing mucosal mass.

Like Starbursts, Tootsie Rolls were less attenuated than most IOFBs and also were variably chewed. When chewed, Tootsie Rolls adhered to mucosal and alveolar surfaces, resembling a plaquelike mucosal lesion (Fig 7).

Bubble Yum was the least attenuating of the IOFBs studied. All of the subjects chewed the gum, so in all of the scans it lacked a characteristic configuration. The interior of the IOFB consistently showed small air locules, which might lead to confusion with an enhancing soft tissue mass or abscess. Bubble Yum might also be mistaken for a cosmetic prosthesis, dentures, or even intraoral hematoma.

Unlike the bubble gum, the hard-shelled gum demonstrated no internal heterogeneity or air locules. Instead, it was usually held in small mucosal recesses. Due to its high attenu- 


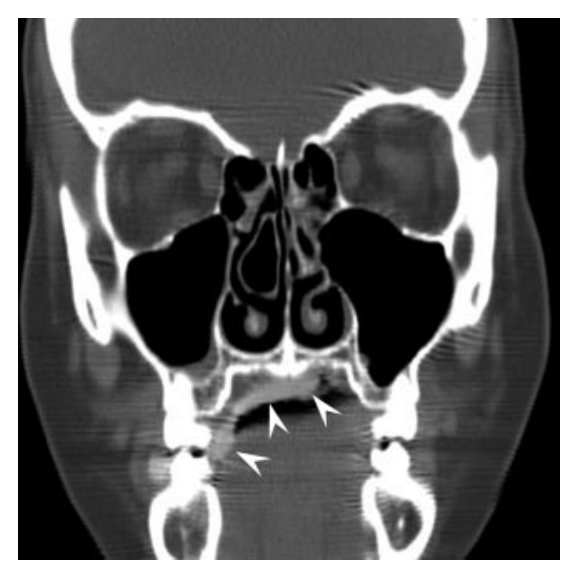

Fig 7. Soft intraoral foreign body. Coronally reformatted CT shows a foreign body as a thin attenuation adherent to the palate (arrowheads). This might be mistaken for an enhancing mucosal mass on an enhanced scan or a different foreign body, such as dentures or a mental implant.

ation, it would probably not be mistaken for a soft tissue mass but more likely a bone tumor or excrescence.

\section{Discussion}

This study describes the imaging characteristics of IOFBs and identifies characteristics that distinguish IOFBs from true intraoral pathology. In general, softer and smaller IOFBs tend to be more easily confused with pathology. The size, shape, and stage of consumption are also important predictors of imaging appearance. Contrast-enhanced scans were more likely to allow confusion of IOFBs with pathology, because the high attenuation of the foreign body could be mistaken for enhanced soft tissue.

The differential diagnosis of the IOFBs is extensive. Examples shown include calculi, hematomas, vascular malformations, abscesses, prostheses, and bony malformations. In addition to those IOFBs that were used in this study, other IOFBs may be encountered. For example, retained chewing tobacco is typically located in the gingivo-buccal sulcus. It has a heterogeneous texture that may resemble gas from a traumatic injury or soft tissue abscess (Fig 8). Although some misdiagnoses (such as mistaking an IOFB for dentures or another prosthetic device) would be unlikely to prompt inappropriate patient care, misdiagnoses such as calculi or abscesses could be important errors.

There are several limitations to this study. A major confounding predictor of the appearance of IOFBs is the degree of consumption. In an actual clinical setting, some of the hard IOFBs may present with smaller size or an unpredictable con-

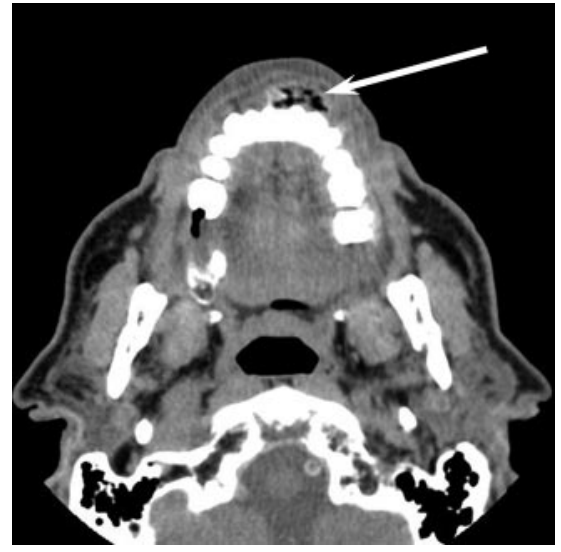

Fig 8. Chewing tobacco. Unenhanced axial CT shows a mass (arrow) of mixed tissue and air attenuation. There is a rim of denser material. On an enhanced scan, this might be mistaken for facial abscess or traumatic injury.

figuration, increasing the likelihood of misdiagnosis. Another confounder is the potential for IOFBs to be broken up into smaller pieces during consumption. Although we selected representative examples of different types of IOFBs, individual IOFBs would probably have specific imaging characteristics that might differ from the examples shown in this study.

In conclusion, intraoral foreign bodies may be easily mistaken for pathologic masses on CT. Smaller foreign bodies and enhanced scans increase the likelihood of such an error. Radiologists should be familiar with the expected appearance of comestible and chewed intraoral foreign bodies to avoid misdiagnosis.

Authors' Note. A complete set of images, showing examples of every type of IOFB, along with examples of true pathology that might be confused with IOFBs, is available at: http://www.pitt.edu/ caram/candygram.htm

\section{References}

1. Ufberg JW, Lex J. Abdominal calcifications on unenhanced CT scan due to gummy bear ingestion. J Emerg Med 2005;28:469-70

2. Geller E, Smergel EM. Bubble gum simulating abdominal calcifications. Pediatr Radiol 1992;22:298-99

3. Bodne D, Quinn SF, Cochran CF. Imaging foreign glass and wooden bodies of the extremities with CT and MR. J Comput Assist Tomogr 1988;12:608-11

4. Gor DM, Kirsch CF, Leen J, et al. Radiologic differentiation of intraocular glass: evaluation of imaging techniques, glass types, size, and effect of intraocular hemorrhage. AJR Am J Roentgenol 2001;177:1199-203

5. Ho VT, McGuckin JF Jr, Smergel EM. Intraorbital wooden foreign body: CT and MR appearance. AJNR Am J Neuroradiol 1996;17:134-36

6. Lin CJ, Su WF, Wang CH. A foreign body embedded in the mobile tongue masquerading as a neoplasm. Eur Arch Otorhinolaryngol 2003;260:277-79

7. Tseng E, Woolley AL. Foreign body simulating a hard palate lesion in a child Int J Pediatr Otorhinolaryngol 1996;38:169-74 